\title{
Unified analytic representation of physical sputtering yield
}

\author{
R.K. Janev ${ }^{\text {a,b }}$, Yu.V. Ralchenko ${ }^{\text {a,c }}$, T. Kenmotsu ${ }^{\text {a,*, }}$ K. Hosaka ${ }^{\text {a }}$ \\ a National Institute for Fusion Science, Data and Planning Center, Graduate University for Advanced Studies, 322-6 Oroshi-cho, \\ Toki-shi, Gifu 509-5292, Japan \\ ${ }^{\mathrm{b}}$ Macedonian Academy of Sciences and Arts, P.O. Box 482, Skopje 91000, Macedonia \\ ${ }^{\mathrm{c}}$ Faculty of Physics, Weizmann Institute of Science, Rehovot 76100, Israel
}

\begin{abstract}
Generalized energy parameter $\eta=\eta(\varepsilon, \delta)$ and normalized sputtering yield $\tilde{Y}(\eta)$, where $\varepsilon=E / E_{\mathrm{TF}}$ and $\delta=E_{\mathrm{th}} / E_{\mathrm{TF}}$, are introduced to achieve a unified representation of all available experimental and sputtering data at normal ion incidence. The sputtering data in the new $\tilde{Y}(\eta)$ representation retain their original uncertainties. The $\tilde{Y}(\eta)$ data can be fitted to a simple three-parameter analytic expression with an rms deviation of $32 \%$, well within the uncertainties of original data. Both $\eta$ and $\tilde{Y}(\eta)$ have correct physical behavior in the threshold and high-energy regions. The available theoretical data produced by the TRIM.SP code can also be represented by the same single analytic function $\tilde{Y}(\eta)$ with a similar accuracy. (C) 2001 Elsevier Science B.V. All rights reserved.
\end{abstract}

Keywords: Sputtering yield

\section{Introduction}

Starting with the pioneering work of Bohdansky [1], several attempts [2-6] have been made to derive a sufficiently simple and accurate analytic representation of either the experimental data [1-4], or the data generated by the simulation codes TRIM.SP [2,3] and ACAT [4], or the combined sets of data [4-6] for the sputtering yield at normal ion incidence. In [2-4], modifications of the original Bohdansky semi-empirical formula [1] were proposed to achieve a better representation of the data (to reduce the rms deviation). In [5] a new formula was proposed which accounts explicitly for the contribution of the electronic stopping power to the sputtering yield, while in [6] the revised Bohdansky formula of [2,3] was modified to achieve a better representation of the data mainly in the threshold region (by using two additional fitting parameters). The major motivation for these efforts has been to present the data in formats appropriate for their implementation in various more complex application codes and to provide a basis for estimation of

\footnotetext{
${ }^{*}$ Corresponding author. Tel.: +81-572-58-2257; fax: +81572-58-2628.

E-mail address: kenmotsu@nifs.ac.jp (T. Kenmotsu).
}

sputtering yield for ion-solid combinations for which neither experimental nor calculated data are available (by using certain well-prescribed interpolation procedures). A common feature of all so far proposed analytic representations of the sputtering yield $Y$ at normal ion incidence is that they use two independent reduced energy parameters, $E / E_{\mathrm{th}}$ and $E / E_{\mathrm{TF}}$, where $E_{\mathrm{th}}$ is the threshold energy and $E_{\mathrm{TF}}$ is the Thomas-Fermi energy. The parameters appear in a natural way, since in the threshold region the behavior of $Y$ is determined by the ratio $E / E_{\mathrm{th}}$, while at high collision energies, where $Y$ is proportional to the nuclear stopping cross-section, the behavior of $Y$ predominantly depends on the reduced energy $\varepsilon=E / E_{\mathrm{TF}}$ only. On the other hand, both TRIM.SP and ACAT Monte Carlo simulation codes are based essentially on the classical binary encounter approximation (with some provisions for the simultaneous (TRIM.SP) and multiple (ACAT) collisions), and their good success in describing the experimental observations indicates that the same classical mechanics laws adequately describe the collisional processes involved in the sputtering phenomenon both in the threshold and highenergy regions. Since there are no fundamentally different physical processes, or mechanisms, governing the sputtering process at low and high collision energies, and the Thomas-Fermi and threshold energies, $E_{\mathrm{TF}}$ and $E_{\mathrm{th}}$, 
are only characteristics of the interaction potential and surface binding energy (i.e., again a potential interaction feature), respectively, it can be assumed that there exists a common (or generalized) energy parameter $\eta=$ $\eta\left(E, E_{\mathrm{th}}, E_{\mathrm{TF}}\right)$ which reflects equally well the ratio of dynamical and potential interaction forces in the entire region of its variation. Of course, in the threshold and high-energy region, $\eta$ should smoothly go over to its proper physical form (i.e., be proportional to $E / E_{\mathrm{th}}$ and $E / E_{\mathrm{TF}}$, respectively).

The purpose of the present work is to find the form of the generalized energy parameter $\eta$ and introduce a normalized sputtering yield $\tilde{Y}$ which will depend on $\eta$ only.

\section{Derivation of $\tilde{Y}$ representation of sputtering yield}

Starting with the expression for the Thomas-Fermi energy $E_{\mathrm{TF}}$ in terms of the masses $M_{1}$ and $M_{2}$ and charges $Z_{1}$ and $Z_{2}$ of the projectile and target atom, respectively, [2,3]

$E_{\mathrm{TF}}(\mathrm{eV})=30.74 \frac{M_{1}+M_{2}}{M_{2}} Z_{1} Z_{2}\left(Z_{1}^{2 / 3}+Z_{2}^{2 / 3}\right)^{1 / 2}$,

we introduce the notation

$\varepsilon=\frac{E}{E_{\mathrm{TF}}}, \quad \delta=\frac{E_{\mathrm{th}}}{E_{\mathrm{TF}}}$

and define $\eta\left(E, E_{\mathrm{th}}, E_{\mathrm{TF}}\right)$ in the form

$\eta=a\left(\frac{\varepsilon}{\delta}-1\right)+b\left[\left(\frac{\varepsilon}{\delta}\right)^{\gamma}-1\right]+1$,

where $a, b$ and $\gamma$ depend on $\delta$. For the realistic collision pairs, the parameter $\delta$ takes values in the range $\sim 10^{-1}-10^{-6}$ (see, e.g., $[2,3]$ ). We further introduce the normalized sputtering yield by the expression

$\tilde{Y}=\frac{Y(\varepsilon, \delta)}{Q G(\delta)}$,

where $Q$ is defined by the relation $Y^{>}=Q s_{n}(\varepsilon), Y^{>}$being the yield in the high-energy region. In [2,3] $Q$ was treated as a fitting parameter of the revised Bohdansky formula (which calculates $s_{n}(\varepsilon)$ on a basis of the $\mathrm{Kr}-\mathrm{C}$ potential instead of using the Thomas-Fermi potential, as was done in [1]). We note that in [2,3] also $E_{\text {th }}$ was treated as a fitting parameter when parametrizing the experimental as well as the TRIM.SP data. The unknown function $G(\delta)$ in Eq. (4) is determined by the requirement that all normalized yields $\tilde{Y}$ have equal maximum value. Such normalized yields have similar shapes as function of $\varepsilon$, but are mutually significantly displaced in the $\varepsilon$-scale due to the different values of their $\delta$-parameters. The requirement that all normalized yields coincide with the normalized yield function having $\delta=1$ gives the dependencies of parameters $a, b$ and $\gamma$ on $\delta$. These dependencies, as well as that for $G(\delta)$, can be fitted with a very high accuracy to the expressions:

$$
\begin{aligned}
& a(\delta)=1.265 \delta\left(0.18+\delta^{2 / 3}\right)^{-1}, \\
& b(\delta)=20.5 \delta^{2 / 5}(1+112 \delta)^{-1}, \\
& \gamma(\delta)=0.81\left(0.0051+\delta^{4 / 5}\right)\left(0.013+\delta^{3 / 5}\right)^{-1}, \\
& G(\delta)=0.85+4.0 \exp \left(-2.94 \delta^{3 / 5}\right) .
\end{aligned}
$$

Transformations (3) and (4), with the implicit dependence of $\tilde{Y}$ and $\eta$ on $\delta$, give the unified representation of the sputtering yield in the form $\tilde{Y}=\tilde{Y}(\eta)$. We note that with the above $\delta$-dependencies of $a, b$ and $\gamma, \eta \rightarrow 1$ when $\varepsilon / \delta=E / E_{\mathrm{th}} \rightarrow 1$, and $\eta \sim E / E_{\mathrm{TF}}$ when $E$ becomes sufficiently large.

In order to demonstrate the quality of the unified $\tilde{Y}(\eta)$ representation of sputtering yield, we show in Fig. 1 all the experimental and TRIM.SP data from $[2,3]$ in the unscaled representation $Y=Y(E)$. This figure shows that for a given value of the impact energy, the $Y(E)$ data for various projectile-target combinations extend over a range of five orders of magnitude. In Fig. 2 we show the same data in the unified (scaled) $\tilde{Y}(\eta)$ representation. It is apparent from this figure that despite the large dispersion of the experimental data (even for a given ion-solid combination and same energy), all data tend to follow a single $\tilde{Y}(\eta)$ line. It should be noted that the transformations (3) and (4) of original data do not affect their uncertainties (i.e., mutual differences), and their original dispersion remains conserved in the $\tilde{Y}(\eta)$ plot.

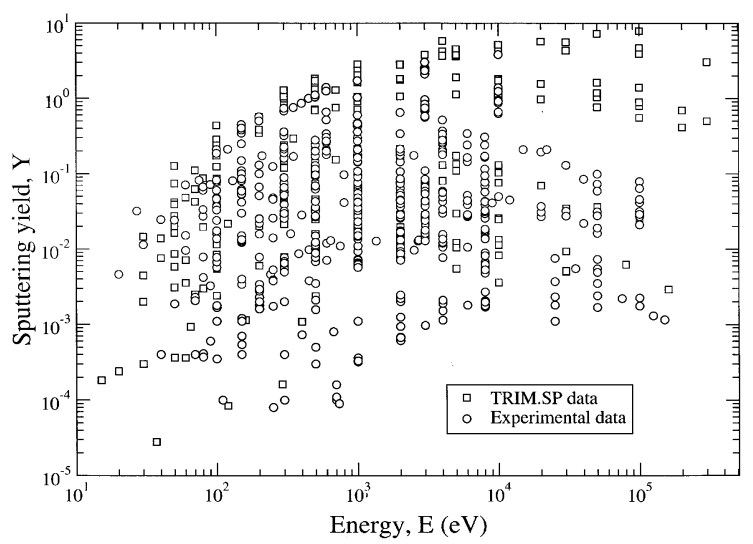

Fig. 1. Experimental and theoretical (TRIM.SP) data from $[2,3]$ in the unscaled $Y(E)$ representation. 


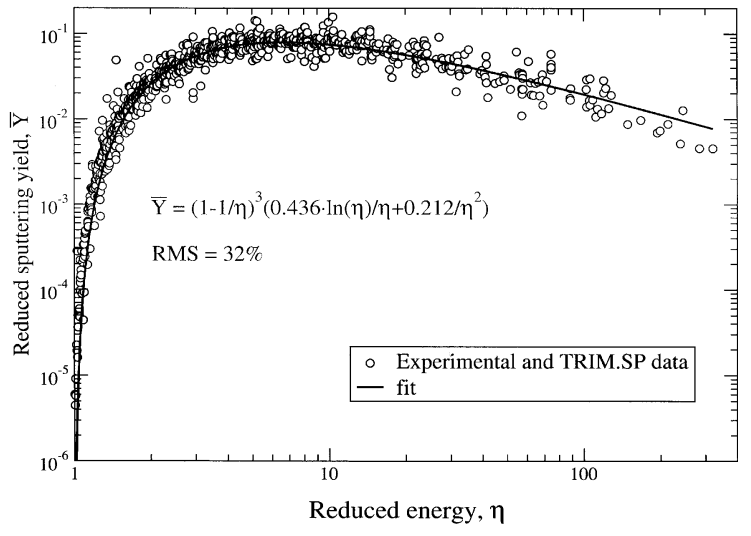

Fig. 2. Experimental and theoretical (TRIM.SP) data from $[2,3]$ in the scaled $\tilde{Y}(\eta)$ representation.

\section{Analytic representation of $\tilde{\boldsymbol{Y}}(\eta)$}

An analytic function representing the scaled $\tilde{Y}(\eta)$ data in the entire $\eta$-region should obviously possess the following properties:

$\eta \rightarrow 1: \tilde{Y}(\eta) \rightarrow \varphi(\eta) \rightarrow 0$,

$\eta \rightarrow \infty: \tilde{Y}(\eta) \rightarrow f(\eta) \sim \frac{\ln \eta}{\eta}$.

The property (10) reflects the asymptotic behaviour of inelastic energy losses at high energies, whereas Eq. (9) is imposed by the existence of energy threshold for the sputtering process. There is, at present, no clear physical basis which allows one to determine the form of the function $\varphi(\eta)$. The condition (9) can be satisfied with many choices of the form of $\varphi(\eta)$ which ensure a fast decrease of $\tilde{Y}(\eta)$ towards the threshold, $\eta=1$. After exploring different possibilities for $\varphi(\eta)$ and $f(\eta)$, we have concluded that the function

$\tilde{Y}(\eta)=\left(1-\frac{1}{\eta}\right)^{\alpha}\left[\frac{A \ln \eta}{\eta}+\frac{B}{\eta^{2}}\right]$ has the simplest and most compact form which satisfies the conditions (9) and (10) and provides equally good fit to the data as the other trial functions with much larger number of free parameters. The best least-square fit of all experimental and theoretical data in Fig. 2 with an rms deviation of $32 \%$ is obtained with the values of parameters:

$\alpha=3, \quad A=0.436, \quad B=0.212$.

The solid curve in Fig. 2 represents this best fit.

\section{Conclusions}

In the present work we have derived a unified analytic representation of the sputtering yield for normal ion incidence which describes the experimental and TRIM.SP data with an accuracy well within their inherent uncertainty. The analytic formula (11) for the normalized sputtering yield can be used to calculate the sputtering yield $Y(E)$ for any ion-monoatomic solid combination for which sputtering data are not available. The required input information to this aim are the corresponding values of $Q$ and $E_{\text {th }}$ which can be obtained by using their semi-empirical expressions (as functions of other ion-solid parameters) given in $[2,3]$.

\section{References}

[1] J. Bohdansky, Nucl. Instrum. and Meth. B 2 (1984) 587.

[2] W. Eckstein, C. Garcia-Rosales, J. Roth, W. Ottenberger, Institute for Plasma Physics (Garching), Report IPP 9/82, 1993.

[3] C. Garcia-Rosales, W. Eckstein, J. Roth, J. Nucl. Mater. 218 (1995) 8.

[4] E.W. Thomas, R.K. Janev, J. Botero et al., International Atomic Energy Agency Report INDC(NDS)-287, 1993.

[5] Y. Yamamura, H. Tawara, At. Data Nucl. Data Tables 62 (1996) 149.

[6] W. Eckstein, in: International Atomic Energy Agency Report INDC(NDS)-410, 1999. 